\title{
Le calendrier mythique chez les Songhay-Zarma
} (Niger)

The mythological calendar of the Songhai-Zarma (Niger)

Jean Rouch

\section{(2) OpenEdition}

\section{Journals}

Édition électronique

URL : http://journals.openedition.org/span/134

DOI : 10.4000/span. 134

ISSN : 2268-1558

Éditeur

École pratique des hautes études. Sciences humaines

\section{Édition imprimée}

Date de publication : 1 septembre 1975

Pagination : 52-62

ISSN : 0294-7080

\section{Référence électronique}

Jean Rouch, «Le calendrier mythique chez les Songhay-Zarma (Niger) », Systèmes de pensée en Afrique noire [En ligne], 1 | 1975, mis en ligne le 03 juillet 2013, consulté le 30 avril 2019. URL : http:/ journals.openedition.org/span/134 ; DOI : 10.4000/span.134 
LE CALENDRIER MYTHIQUE CHEZ LES SONGHAY-ZARMA (NIGER)

par Jean ROUCH

"Puisque je vais parler des divinités du culte de possession ( les holey) et de leur répartition spatio-temporelle, je commence par orienter le pays songhay suivant le système nigérien, c'est à dire par rapport au fleuve Niger : ainsi, l'Est est appelé le hausa, l'Ouest le gurma, le Nord danga ( "l'amont" ou azawak = l'Air ), et le sud dendi ( le "fil de l'eau"). Cette orientation par le fleuve est assez particulière puisque à Tombouctou, par exemple, le hausa devient le Nord, le gurma le sud. Elle varie donc par rapport aux points cardinaux en fonction de l'endroit oì l'on se trouve. Ceci, pour moi, attesterait que les Songhay sont originaires de la région du double $V$ où 1 'on trouve effectivement des groupes hausa et gurma à l'Est et à l'Ouest du fleuve, et qu'ils ont transporté ensuite leur système d'orientation jusqu'à Djenné, où l'ouest devient le hausa et l'Est le gurma bien qu'il n'ait aucun groupe hausa ou gourmantché dans cette région.

La langue songhay $n^{\prime a}$ aucune connection avec les langues voisines: malinké-bambara, voltaiques ou hausa. Les seules corrélations trouvées jusqu'à présent par Greenberg seraient avec des groupes de l'affluent du Niger, la Bénoué, du Nord-Est de la Nigéria. Ceci rejoindrait la vieille théorie de L. Homburger faisant venir les pêcheurs sorko de Haute Egypte en suivant la Bénoué puis en remontant le Niger par étapes jusqu'au lac Débo où ils se seraient heurtés aux "maItres de l'eau", les Bozo.

1) Les pêcheurs sorko et le mythe de Faran Maka, leur ancetre.

Ces maItres de l'eau étaient les "martres des biefs", alliés aux divinités locales du fleuve et des mares. Ce sont des do, 'da ou dali.

Le mythe songhay raconte comment toutes ces petites chefferies locales ont été remplacées par une chefferie unique, assumée pax les Sorko, pêcheurs du Niger, alliés au génie de 1 'eau. Le mythe raconte aussi comment Harakoy Diko, génie de l'eau, a rendu captifs les gênies de lieu de ces biefs dont le 
culte était assuré par les do. Aịnsi, pour moi, les Bozo étaient des do, deg:"unatres áe biefs", mais ce ne sont pas les "maftres du fleuve", comme les sorko,qui l'occupent depuis la Bénoué jusqu'à Mopti. Les Sorkawa (groupe sorko de la rivière Kebbi), pêcheurs itinérants, partaient de Nigéria et pêchaient jusqu'à Mopti sur leur fleuve jusqu'au moment où les autơitếs maliennes les ont refoulêes comme "êtrangers".

Les sorko ont donc toujours la notion d'un fleuve unique comprenant un ensemble discontinu de biefs sur lesquels les "maitres de l'eau"ont conservé tout leur pouvoir local : les do ont droit à une part des captures des Sorko. Sans ce don rituel, les do peuvent "gâter" le fleuve, causer des accidents. Et $s i$ une de leurs pirogues coule, les Sorko doivent s'adresser au do du bief qui fait les sacrifices et qui va chercher la pirogue.

A la différence des Bozo et des Sorkawa, les Sorko sont essentiellement des pêcheurs au harpon, ils n'utilisent presque jamais les filets. Ce sont en fait des chasseurs traditionnels de lamentins, de crocodiles et d'hippopotames.

D'après le mythe songhay, Gao a été fondé par Faran Maka, l'ancêtre des Sorko ( les traditions écrites rappellent. que Gao a été fondé au IXème siècle ).

Le pêcheur Faran Maka est considéré comme étant né dans le " $W$ " d'un père sorko et d'une mère appelée Maka, génie de lieu (actuellement encore responsable du "W"). Faran fonda Gao, se battit contre les Bella qu'il chassa hors du fleuve ou ils étaient des hippopotames. (1). Faran fonda ensuite sarafere dans le delta central du Niger, puis revint mourir à Bamba Sorko ( au nord de la boucle du fleuve).

Ce personnage historique et mythique est réputé avoir été le premier initié aux danses de possession. Les divinités

(1) Les Bella sont aujourd'hui les captifs des Touareg, mais leur noyau originel aurait été un groupe d'hippopotames (alliance totémique?), originaire de la région de GaoTombouctou, vaincu d'abord par les sorko et ensuite conquis par les Touareg. 
du fleuve ( tooru), alliées à Faran Maka, luttant contre les génies locaux ( $z i n), l$ 'appelèrent au secours. Au terme d'un combat dans le pays gurma, Faran Maka transforma le chef des $z i n$ en harpon, qu'il saisit pour tuer un hippopotame. En reconnaissance, les tooru, libérés et devenus maitres du fleuve, le designèrent comme prêtre principal de leur culte et lui apprirent à initier les hommes à la danse de possession.

Lorsque la paix fut revenue parmi les génies, l'un des plus turbulents, Dongo, monta au ciel et utilisa pour la première fois un pouvoir hérité de son père : il tua par la foudre des villageois. Ses frères et sa mère adoptive, Harakoy Diko, convoquèrent Faran Maka et lui dirent: "Nous allons te montrer ce qu'il faut faire pour éviter que Dongo foudroie les hommes et pour qu'il leur donne la pluie". C'est ainsi qu'eut lieu la première cérémonie ( le yenendi - "refroidir") au cours de laquelle les danseurs furent possédés par les divinités tooru. Au premier yenendi, les dieux vinrent eux-mêmes et enseignèrent aux hommes les airs de musique, les louanges et les pas de danse nécessaires. I'année suivante, Faran Maka organisa, le septième mois de la saison sèche, le deuxième yenendi au cours duquel les hommes et les dieux possédant leur "chevaux" passèrent le contrat de l'année à venir. Et depuis, les yenenäi furent toujours cêlébrês par les sorko, prêtres-pêcheurs descendant de 1 'ancêtre Faran Maka.

Ainsi, dès le départ, tout est axé sur le fleuve et sur le groupe prédominant des Sorko.

Sur le plan historique, il est certain que le fleuve a joué un rôle considérable dans tout le développement de l'histoire du Songhay et a été l'élément irremplaçable que n'avaient pas à leur disposition le Mali ou le Ghana. L'un des principaux dignitaires de l'empire songhay, le chef des piroguiers (dikoy), était un Sorko. Les songhay ont fait la guerre en utilisant d'une part les cavaliers, d'autre part les pêcheurs et leurs pirogues. Lor̀sque les Marocains sont arrivês à Gao; en 1591, toute la population est partie en pirogue; les gens se sont réfugiés en suivant le fleuve, au fil de l'eau, au Dendi. 
2) Le mythe des divinités magistrales du fleuve et du ciel, les tooru.

Le terme tooru, peut-être d'origine arabe, veut dire "idole", c'est à dire autel sur lequel on fait des sacrifices, qui est doué de pouvoir et qui parle.

Les tooru, divinités magistrales des songhay-zarma, ont la particularité d'être formés des représentants de toutes les ethnies qui participèrent à l'élaboration de l'empire songhay. Ils sont les descendants d'une femme peule qui vivait dans une Ile du "W" à Gambou, sous sept cailcedrats, et qui divorçait de ses maris au fur et à mesure qu'ils lui faisaient des enfants. Elle eut ainsi les principaux tooru :

- un Songhay du Nord : Kyirey

- un Touareg

: Mahama

- un Gourmantché

: Moussa

- un Hausa de Yawri

: Manda

- un Sorko ou Do

: Faranbarou

- une Peule(ou captive de Peul) : Nayanga

- un Bariba (ou Bella): Dongo

Le Songhay est responsable de la foudre; sa couleur est le rouge. Le Touareg est responsable de la brousse du Nord; sa couleur est le blanc.

Le Gourmantché est responsable des vents; sa couleur est le noir et le blanc.

Le Hausa est responsable de la forge, des pierres de foudre et du "briquet" (éclairs d'altitude); sa couleur est le noir.

Le jeune Sorko est responsable du fleuve (comme adjoint); sa couleur est le blanc et le rouge.

La Peule est responsable du fleuve (avec sa mère et son filleul sorko); sa couleur est le blanc.

Le Bariba (Bella) est responsable du tonnerre et de la pluie; sa couleur est le noir.

Le plus important est le dernier, le Bariba (ou Bella), Dongo. C'est un chasseur d'éléphants; c'est un captif qui a reçu de son père la maftrise du feu. Ce personnage est représenté 
comme un homme fort, vindicatif, bête, désobeissant, mais fidèle. Il est le vrai maitre du feu du ciel et de la pluie, mais il ne peut agir sans l'accord de sa famille et la présence de deux de ses frères au moins.

Les tooru passent quatre mois de l'année dans le ciel, au moment de la saison des pluies. A la fin de cette période, chaque tooru regagne sa région : soit le fleuve, soit la brousse (rive gurma, rive hausa, azawak).

Ainsi, les tooru sont les maîtres des éléments naturels les plus importants pour les Songhay : le fleuve, la foudre, le tonnerre (1), la pluie, le vent.

Voici le système météorologique élaboré par les Songhay. Au début de la saison des pluies, Harakoy Diko, génie du fleuve, réunit ses enfants et leur dit que le temps est venu. Dans le ciel montent alors le Songhay Kyirey, gênie de la foudre, le Gourmantché Moussa, génie du vent, Manda le forgeron hausa, le Bariba Dongo, maftre du tonnerre, et la servante peule Nayanga. Ils installent leur"maison" sur les nuages venus de l'ouest et collectés par le génie du vent, quand, en mars, la mousson de 1'Atlantique pousse les nuages à basse altitude vers l'est. Pendant un ou deux mois, Moussa le Gourmantché conduit ces nuages jusqu'au jour où il demande au vent d'est de plonger vers le sol et àe ramener les nuages accumulés vers 1 'ouest : c'est un grain. Ce vent $d$ 'est est chevauché par tous les tooru dont Nayanga qui porte la pluie dans un grand "canari". Ils avancent sur le vent et Kyirey, l'arné, le guide, désigne avec sa lance l'endroit où Dongo doit frapper. S'il ne voit pas bien, Dongo demande a Manda de sortir son briquet de forgeron pour éclairer: ce sont les éclairs d'altitude annonciateurs d'orage. Puis Dongo prend les pierres que Manda a taillées en forme de haches et, secouant ses vêtements de cuir (grondements du tonnerre), il "bombarde"la cible désignée par l'éclair de Kyirey : c'est la foudre qui met le feu à la brousse et aux villages, qui tue les gens. Derrière lui, Nayanga déverse l'eau du vase de pluie

(1) Les Songhay font une distinction entre la foudre que l'on voit et le tonnerre que l'on entend plus tard. 
pour eteindre le feu. Alnsi, les dieux ont donné aux hoinmes le feu et l'eau du ciel nécessaire aux. récoltes.

La prépondérance de Dongo est basée sur le fait que la foudre tue beaucoup de gens. En 1970, par exemple, dans un rayon de $100 \mathrm{~km}$. autour de Niamey, $11 \mathrm{y}$ eut 120 foudroyés pendant la saison des pluies. Dans les cérémonies de purification de

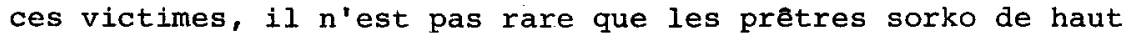
savoir déterrent le projectile meurtrier : une hache de pierre néolithique qui deviendra un talisman contre le feu du ciel.

A la fin de la saison des pluies (octobre), les tooru descendent du ciel, reviennent au fleuve, chez leur mère. Ceci correspond aux crues du fleuve Niger. En effet, celui-ci ne reçoit aucun vrai affluent après Mopti; ses crues sont dues aux pluies qui sont tombées dans les montagnes de Guinée et qui mettent six mois à parvenir jusqu'au pays songhay. Ainsi, . dans cette région, le fleuve est bas en saison des pluies et haut en saison sèche. On comprend donc très bien que les tooru et leur mère se rassemblent en saison sèche dans le fleuve qui est alors en crue, pour l'abandonner quand il baisse, à l'arrivée de la saison des pluies.

3) Les autres familles de génies.

En dehors des tooru du fleuve, il existe d'autres divinités, considẹrées comme secondaires. On distingue :

- les gandji koare : "brousse blanche". Ce sont des Touareg, des gens du Nord. Ils n'ont pas grande influence. Ils sont, à mon sens, le tribut que paye la religion des holey à l'Islam, car beaucoup de ces génies blancs sont des marabouts.

- les gandji bi : "brousse noire". Ce sont des génies gourmantché ou kouroumba, maitres du sol. Ces génies noirs tenaient leurs assises dans une forêt au Nord de Niamey, sur la rive hausa. Ils ont été vaincus par les tooru et sont devenus leurs captifs. - les hargey : les "froids". Ce sont les enfants de Nyaberi (la "grand'mère"), fille du génie de l'eau qui l'a fait chasser du ciel. En tombant, elle s'est cassée les deux jambes et est restée paralysée. Elle est allée vivre dans les cimetières; elle est devenue une sorcière qui mange le double des hommes. 
- les dogwa : génies du hausa, responsables des maladies.

A ces groupes, il faut ajouter les genies "modernes" : - les hauka : génies de la force, de la "colonisation". Ils sont depuis peu rattachês aux tooru et considérés comme les captifs ou les enfants que Dongo aurait eu à la Mecque.

- les sasale : génies lubriques et funèbres apparus au Niger vers 1968 et sur lesquels on ne sait pas encore grand chose.

Ces familles se répartissent les principales activités du monde invisible et représentent un certain nombre de forces de la nature (les tooru etant responsables des forces principales).

Toutes ces divinités sont soumises aux tooru, mais leurs prêtres sont des zima, spécialistes des danses de possession, qui permettent aux hommes d'être en communication presque permanente avec un panthéon de cent cinquante à cent soixante génies.

4) La sécheresse du Sahel et le ni: ine. Soraytioior spatio-temporelle des holey.

En 1968, tout au début de la grande sécheresse, ce sont les tooru qui en ont parlé les premiers. En août, au cours d'une cérémonie faite pour Dongo - qui avait foudroyé le campement peul de Gangel - celui-ci a "refusé le pardon". Il a refusé les sacrifices en proférant des menaces qu'alors personne ne comprenait. Or, il annonçait que les pluies ne viendraient pas parce que les hommes abandonnaient les coutumes. C'était la première fois, alors que le mil était déjà sorti de terre, que s'exprimait publiquement un propos alarmiste au sujet de la récolte à venir. En fait, tous les vrais paysans savaient que 1 'année serait mauvaise, mais personne n'avait osé en parler. Dongo I'avait fait le premier.

Avec Diouldé Laya, Evelyne Poncet, Mamadou Ouattara, nous avons essayé de collecter toutes les informations météorologiques, géographiques, agronomiques et traditionnelles sur la sécheresse. Nos connaissances sont encore rudimentaires. Par exemple, ce $n^{\prime} \in s t$ pas le volume d'eau, mais les chutes de pluie à certains moments de la saison qui permettent d'avoir une bonne récolte. Autre exemple : si les sécheresses anciennes ont 
été moins catastrophiques que celle-ci, cela est du sans doute au fait que, jusqu'en 1956, on cultivait au Niger un mil rustique, remplacé depuis par un mil à haut rendement qui, effectivement, fournit une récolte deux ou trois fois plus abondante quand les conditions climatiques sont bonnes, mais qui ne donne rien lorsque la saison des pluies est mauvaise. En fait, en dehors de l'équipe H. Raulin-N. Echard, nous n'avons jamais fait de calendrier agricole précis. En particulier, nous n'avons jamais étudié, pendant une saison des pluies, ondée après ondée, la corrélation des chutes d'eau et de l'évolution du mil. Personnellement, j'ai essayé, avec Mamadou Ouattare et Moussa Hamidou, d'examiner l'interprétation de ces phénomènes dans le mythe en suivant les consultations permanentes entre les hommes et les divinités responsables. Chaque année, au début de la saison des pluies, on célèbre le yenendi. Durant cette cérémonie, on fait venir toutes les divinités du ciel et les hommes leur demandent comment seront la pluie et la récolte correspondante. Cette cérémonie très importante qui, il y a plusieurs années, avait tenđance à disparaître, a retrouvé une nouvelle vigueur depuis la sécheresse.

Ainsi, d'année en année, s'est manifestée une condamnation indirecte de l'Islam : nous n'avons pas de pluie, il n'y plus de bonnes récoltes depuis que les hommes prient aux dépens des cultes traditionnels. L'Islam est une religion de nomades et non une religion de cultivateurs.

De même, une réaction contre la chefferie s'est manifestée : le chef est le responsable traditionnel de la pluie et de la culture. Si la pluie ne vient pas, c'est parce que le chef ne suit plus les coutumes. Jadis, un chef de canton devait quitter sa chefferie au bout de sept ans s'il y avait des mauvaises récoltes ininterrompues. Mais aujourd'hui, la plupart des chefs ont été nommés par le pouvoir politique; ils se retranchent derrière lui parce qu'ils sont musulmans ou professent un islamisme de façade.

Il y a deux ans - alors que les pluies ont été meilleures dans la région de Niamey - est apparue une chenille qui a mangé les épis de mil. On la connaissait depuis longtemps. Ce qui a 
surpris, c'était l'ampleur de ses ravages. Les agronomes pensent que cette chenille - qui n'aime pas I'eau - a pu se développer pendant les années de sécheresse précédentes. Le village de Simiri où je travaillais, au Nord de Niamey, possède un temple oư a lieu la plus importante cérémonie yenenäi du Niger. Or, après l'arrivée des chenilles, en 1973, ce village a été foudroyé : il était impur. On a appelé le génie du tonnerre et tous les tooru pour leur demander des comptes. Les génies ont accusé le chef de ne pas aller dans les champs pour "manger les chenilles", possédé par le holey du canton. J'ai appris ainsi que chaque canton avait son holey et qu'un vrai chef de canton devait être possédé par ce génie lors de son intronisation. Mais, depuis l'indépendance, presqu'aucun chef de canton n'a été possédé par "honte" de suivre les coutumes. J'ai relevé les noms de ces holey associés aux chefs de cantons et j'ai commencé à en établir la répartition géographique. Par exemple, à Karma c'est Kyirey; à Namaro c'est Serki; à Torodi c'est Dongo; à Ayorou c'est Nyabéri,etc.... Il n'y a apparemment aucune corrélation entre le tooru ou le holey qui est représenté et la région dans laquelle il agit. Par exemple, à Niamey qui est un canton récent, il y a trois holey : Serki qui est hausa, Zambarki qui est doogowa et Mossize qui est mossi.

Je pense que l'établissement d'une carte de ce genre (en tenant compte des holey de village) est le point de départ d'un travail fructueux.

Par ailleurs, en essayant d'étabiir un calendrier agricole précis à la fin de la dernière saison des pluies (1974), avec E. Poncet, D. Laya et $M$. Ouattara, nous avons vu apparaitre un calendrier rituel des holey : les génies sont associés non seulement à des lieux, mais aussi à des saisons.

L'année traditionnelle se divise en quatre saisons au cours desquelles ont lieu des sacrifices et des rites divers. Ces cérémonies font appel à des familles de holey qui se répartissent de la façon suivante en fonction des saisons : a) les tooru ou génies du ciel : la saison des pluies (maioctobre) 
b) les gandji bi ou génies de brousse : la saison mi-froide (heemar), la récolte jisima foori (octobre-décembre)

c) les hargey, fils et filles de la grand'mère Nyaberi, génie du froid : la saison froide jaw (décembre-février)

d) les dogwa ou génies chauds (hausa) : la saison chaude cekonno (février-mai)

e) les hauka, fils de Dongo, apparaissent dans presque toutes les cérémonies.

Voici. le calendrier rituel des holey que nous proposons et qui doit servir de base d'enquête à Mamadou ouattara:

a) Les cérémonies annuelies régulières.

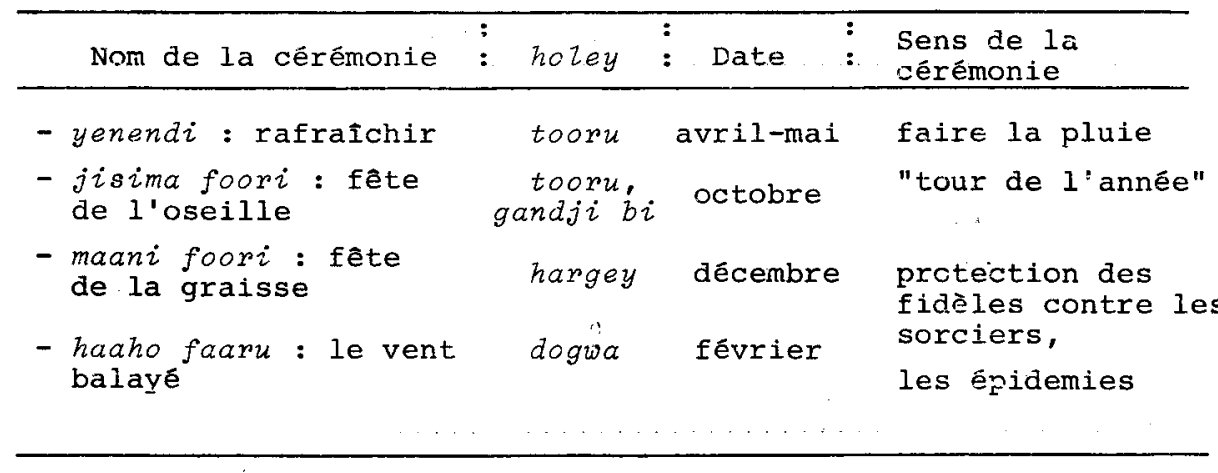

b) Les cérémonies générales. Elles ont lieu chaque année, sans date dêterminée. Ce sont des fêtes de remerciements destinés a tous les holey-(sabuyan : "faire merci").

c) Les cérémonies occasionnelles. Elles ont lieu à partir du premier mois traditionnel (c'est à dire à partir de la dernière pluie) jusqu'au septième mois, date du yenendi. ce sont d'une part les rites de 1'initiation (hoorandi) qui s'adressent à tous les holey et qui durent sept jours, d'autre part les cérémonies exceptionnelles (holey hori : "fête de holey") effectuées en cas de maladies, accidents, famine, sécheresse etc... (durée: de un à trois jours).

Après le yenendi, toutes les cérémonies avec batteries de 
calebasses sont interdites. Ia raison en est que les tooru et principalement Dongo, "refroidis" après le yenendi, ne doivent pas se mettre en colère, sauf évidemment dans les cas très graves (manque de pluie, accidents de foudre, noyades, maladies etc...).

Le premier jour de la saison sèche est celui de la dernière pluie. On calcule les jours qui le séparent de la prochaine lune, on compte les sept mois lunaires, puis on ajoute ou on retranche dix jours. C'est à ce moment-là que doit avoir lieu le yenendi qui ouvre la saison des pluies suivante.

Il n'y a pas de danse de holey pendant le Ramadan. Si le yenendi tombe au moment du Ramadan, on le célèbrera plus tard ( sauf pendant la période des semailles).

Je voudrais dire enfin comment les holey ont réagi à la fin de la sécheresse. Un des zima de Niamey est mort au mois de mai dernier, juste avant le yenendi. A l'occasion de la cérémonie funéraire, les tooru sont venus et Dongo a parlé. Il a dit que ces dernières années avaient été mauvaises à cause de l'indifférence des paysans : au lieu de se cotiser pour célébrer le yenendi, ils attendaient une subvention du gouvernement alors que traditionnellement chaque famille doit participer aux frais. Dongo a rappelé que dans la région de kirkisoy, sur la rive ouest de Niamey, il n'y avait pas eu de famine parce que chacun avait apporté sa contribution. Dongo a rappelé ensuite que les membres de la société des holey - danseurs et fidèles - formaient une seule famille, indépendamment des critères d'origine sociale, de la richesse, du sexe et de l'âge; si tous ces fidèles s'unissaient comme jadis, ils auraient le pouvoir et personne ne pourrait rien contre eux. Ces paroles de Dongo commentaient la récente et importante extension des cultes de possession. Le yenendi suivant (1974) a êté réalisé grâce à quelques contributions personnelles, mais aussi grăce à une subvention du gouvernement; le résultat a été indécis : une bonne rêcolte, mais gâchée par la chenille. L'opinion publique l'a attribuée à la carence des chefs de canton qui ont "la chance de la terre" dans leur bonnet. 